ILL-(TH)-01-10

October 2001

revised, May 2002

\title{
The Maximal Abelian Gauge, Monopoles, and Vortices in SU(3) Lattice Gauge Theory
}

\author{
John D. Stack and William W. Tucker \\ Department of Physics \\ University of Illinois at Urbana-Champaign \\ 1110 W. Green Street \\ Urbana, IL 61801 \\ and \\ Roy J. Wensley \\ Department of Physics and Astronomy \\ Saint Mary's College \\ Moraga, CA 94575
}

\begin{abstract}
We report on calculations of the heavy quark potential in $S U(3)$ lattice gauge theory. Full $S U(3)$ results are compared to three cases which involve gauge-fixing and projection. All of these start from the maximal abelian gauge (MAG), in its simplest form. The first case is abelian projection to $U(1) \times U(1)$. The second keeps only the abelian fields of monopoles in the MAG. The third involves an additional gauge-fixing to the indirect maximal center gauge (IMCG), followed by center projection to $Z(3)$. At one gauge fixing/configuration, the string tensions calculated from MAG $U(1) \times U(1)$, MAG monopoles, and IMCG $Z(3)$ are all less than the full $S U(3)$ string tension. The projected string tensions further decrease, by approximately $10 \%$, when account is taken of gauge ambiguities. Comparison is made with corresponding results for $S U(2)$. It is emphasized that the formulation of the MAG is more subtle for $S U(3)$ than for $S U(2)$, and that the low string tensions may be caused by the simple MAG form used. A generalized MAG for $S U(3)$ is formulated.
\end{abstract}

PACS Indices: 11.15.Ha 12.38.Gc 


\section{Introduction}

One of the most important ideas in the theory of quark confinement is the notion of abelian projection, introduced long ago by ' $t$ Hooft [1]. The basic conjecture made by 't Hooft was that in an appropriate partially fixed gauge, confinement can be understood for $S U(N)$ pure gauge theory by retaining only the gauge fields belonging to the maximal abelian subgroup $U(1)^{N-1}$. In 't Hooft's original paper, there were several suggestions for how to partially fix the gauge, leaving the maximal abelian subgroup unfixed. Subsequent work in $S U(2)$ lattice gauge theory has focussed on the well known 'maximal abelian gauge' (MAG). The main purpose of the present work is to perform lattice gauge theory calculations which test 't Hooft's idea of abelian projection for $S U(3)$, using the simplest possible form of the MAG.

\subsection{Beyond Abelian Projection}

The projection process can be carried beyond abelian projection, keeping a still smaller set of degrees of freedom. Closely related to abelian projection or abelian dominance is the postulate of monopole dominance. This assumes that the confining physics in the abelian gauge fields originates in the magnetic current of monopoles. The monopoles are assumed to be similar in nature to 't Hooft-Polyakov monopoles, moving on world lines. It is known that a dense network of magnetic current is the agent of confinement in $U(1)$ lattice gauge theory [2]. The key question is whether a corresponding network can explain confinement in $S U(N)$ lattice gauge theory.

Another possibility is that the confining degrees of freedom are really associated with the $Z(N)$ center of $S U(N)$. Since $Z(N) \subset U(1)^{N-1}$, this can be studied after making an abelian projection to $U(1)^{N-1}$. After the initial gauge-fixing and abelian projection, the $U(1)^{N-1}$ abelian links are subjected to a second gauge-fixing and projection, leaving a set of $Z(N)$ or center-valued links. This is the 'indirect maximal center gauge' (IMCG). The IMCG uses the MAG as an intermediate stage, as opposed to the 'direct maximal center gauge' (DMCG), which does not. For either case, center dominance is tested by using $Z(N)$ links, or closed world sheets of plaquettes carrying $Z(N)$ flux. The latter are called $(\mathrm{P})$-vortices.

\subsection{Gauge Ambiguities and $\mathrm{SU}(2)$ vs. $\mathrm{SU}(3)$}

In this paper, we work exclusively with 'maximal' gauges; gauges in which a certain functional of the links is maximized before the links are abelian or center-projected. The use of a maximal gauge has an intuitive appeal. In the case of abelian projection, if the charged degrees of freedom are to be deleted, why not first transform to a gauge where they are made as small as possible? Maximal gauges also have the desirable practical feature that the noise level in projected configurations is quite low.

A complication associated with maximal gauges is the presence of gauge or Gribov ambiguities. The functionals used have many local maxima for a given configuration. Each 
local maximum of the gauge functional is a 'gauge copy', and the results of numerical calculations depend on which gauge copy is chosen for each configuration. That this variation with gauge copies occurs at all is caused by projection. If the complete set of gauge degrees of freedom is kept, a gauge invariant quantity like a Wilson loop has the same value at any point on the gauge orbit of a configuration. But if certain degrees of freedom are deleted, then the value of the projected Wilson loop will in general be different at different points on the orbit, even if the chosen points are local maxima of a gauge functional.

$S U(2)$ calculations have shown a clear qualitative trend for this variation due to gauge copies. Gauge copies with higher values of the functional produce projected configurations which are more ordered and produce lower string tensions. This is true for the maximal abelian gauge followed by abelian projection, as well as the direct maximal center gauge followed by center projection. In both cases, if for each configuration a randomly chosen gauge copy is used, the projected string tension turns out to be larger than the full $S U(2)$ result. If instead, a collection of gauge copies is generated for each configuration, selecting a subset and then picking the member of the subset with the highest value of the gauge functional leads to a reduced string tension. For the MAG and abelian projection, recent work suggests that use of the 'best' (global maximum) gauge copy for each configuration would lead to an abelian string tension quite close to the full $S U(2)$ value [3]. For the DMCG and center projection, there is more variation. As gauge copies with increasing values of the center gauge functional are used, the $Z(2)$ string tension decreases from an initial value above the full $S U(2)$ string tension to a value well below it [4]. While the global maximum of the gauge functional evidently plays a different role in the two projection schemes, for the purposes of this paper, the important point is that for both abelian and center projection, there exist gauge copies that give string tensions very close to the exact $S U(2)$ value.

For $S U(3)$, the correct formulation of the MAG condition is a more subtle matter than it is for $S U(2)$. This is discussed in detail in Sec.(22). The fact that there is more than one possible form for the MAG is important, since one of our main results is that for the simplest form of the MAG in $S U(3)$, it is practically certain that no gauge copies exist which produce a $U(1) \times U(1)$ string tension close to the full $S U(3)$ value.

Maximal center gauge conditions are discussed in Sec.(3). In our numerical work, we used the IMCG which has the MAG as a preliminary step. Here we also find a low string tension. The amount by which the $Z(3)$ string tension is low depends on the treatment of gauge copies. Since the MAG and abelian projection are an intermediate stage, the low values we find for the $Z(3)$ string tension are not necessarily a problem of center projection in $S U(3)$, but may be reflecting the difficulty discussed above for the simple form of the MAG.

Our numerical calculations were performed at $\beta=5.9$ on a $10^{3} \times 16$ lattice and at $\beta=6.0$ on a $16^{4}$ lattice. The full $S U(3)$ results are in Sec.(4). For the MAG, the $U(1) \times U(1)$ results are in Sec.(5), and monopole results are in Sec.(6). For the IMCG, the $Z(3)$ results are in Sec.(7). We find that as in $S U(2)$, the monopole and center vortex degrees of freedom are intimately connected. At $\beta=6.0$, for both the MAG and IMCG, 
we investigated the dependence on gauge copies. We confirm for both gauges, that gauge copies with higher values of the gauge functional lead to lower string tensions. This is the same general behavior as seen in $S U(2)$. Sec.(8) contains a summary and conclusions.

\section{Maximal Abelian Gauge}

In this section, we discuss the maximal abelian gauge condition for $S U(3)$, comparing to the familiar case of $S U(2)$. For $S U(2)$, we regard $A_{\mu}^{3}$ as the abelian field, and by making gauge transformations, suppress the 'charged' fields $A_{\mu}^{1}$ and $A_{\mu}^{2}$ to the greatest extent possible. The continuum functional

$$
G_{m a g}^{s u 2}=\int d^{4} x\left[\left(A_{\mu}^{1}\right)^{2}+\left(A_{\mu}^{2}\right)^{2}\right]
$$

is minimized over $S U(2)$ gauge transformations. Near a minimum, $G_{m a g}^{s u 2}$ is stationary when the gauge fields are varied by an infinitesimal gauge transformation,

$$
A_{\mu}^{a} \rightarrow A_{\mu}^{a}-\partial_{\mu} \theta^{a}+\epsilon^{a b c} \theta^{b} A_{\mu}^{c}
$$

Since $G_{m a g}^{s u 2}$ is invariant to a small abelian gauge transformation involving $\theta^{3}$, only transformations involving $\theta^{1}, \theta^{2}$ lead to gauge conditions. If we combine the gauge fields into charged combinations, $A_{\mu}^{ \pm}=\left(A_{\mu}^{1} \pm i A_{\mu}^{2}\right) / \sqrt{2}$, the conditions can be written as

$$
\partial_{\mu} A_{\mu}^{ \pm} \pm i A_{\mu}^{3} A_{\mu}^{ \pm}=0
$$

Turning to $S U(3)$, there are now two abelian gauge fields, $A_{\mu}^{3}$ and $A_{\mu}^{8}$. The remaining six gauge fields are all charged with respect to at least one of $A_{\mu}^{3}, A_{\mu}^{8}$. Treating these charged fields democratically, the simplest maximal abelian gauge condition for $S U(3)$ would minimize the functional

$$
G_{\text {mag }}^{s u 3}=\int d^{4} x\left[\left(A_{\mu}^{1}\right)^{2}+\left(A_{\mu}^{2}\right)^{2}+\left(A_{\mu}^{4}\right)^{2}+\left(A_{\mu}^{5}\right)^{2}+\left(A_{\mu}^{6}\right)^{2}+\left(A_{\mu}^{7}\right)^{2}\right]
$$

over the $S U(3)$ gauge group. An infinitesimal $S U(3)$ gauge transformation is

$$
A_{\mu}^{a} \rightarrow A_{\mu}^{a}-\partial_{\mu} \theta^{a}+f^{a b c} \theta^{b} A_{\mu}^{c}
$$

where $f^{a b c}$ are the $S U(3)$ structure constants. Since $G_{m a g}^{s u 3}$ is invariant to a small abelian gauge transformation involving $\theta^{3}$ and $\theta^{8}$, there are six gauge conditions arising from the remaining six angles. These conditions can easily be expressed in terms of the three $S U(2)$ subgroups of $S U(3)$. We denote the three subgroups and their gauge fields as follows:

$$
\begin{array}{cl}
I: & A_{\mu}^{1}, A_{\mu}^{2}, A_{\mu}^{3} \\
V: & A_{\mu}^{4}, A_{\mu}^{5}, A_{\mu}^{3 \prime} \\
U: & A_{\mu}^{6}, A_{\mu}^{7}, A_{\mu}^{3 \prime \prime},
\end{array}
$$


where $A_{\mu}^{3 \prime}=\frac{1}{2} A_{\mu}^{3}+\frac{\sqrt{3}}{2} A_{\mu}^{8}, A_{\mu}^{3 \prime \prime}=-\frac{1}{2} A_{\mu}^{3}+\frac{\sqrt{3}}{2} A_{\mu}^{8}$, and with some abuse of notation, quark model terminology has been used to denote the subgroups. Consider the response of $G_{m a g}^{\text {su3 }}$ to an infinitesimal gauge transformation in the $I$-spin subgroup. Only the terms in $G_{m a g}^{\text {sua }}$ involving $A_{\mu}^{1}, A_{\mu}^{2}$ vary under this transformation, so demanding that $G_{\text {mag }}^{\text {su3 }}$ be stationary leads to Eq.(3) again. Likewise, demanding that $G_{m a g}^{s u 3}$ be stationary with respect to a $V$-spin gauge transformation leads to

$$
\partial_{\mu} A_{\mu}^{ \pm \prime} \pm i A_{\mu}^{3 \prime} A_{\mu}^{ \pm \prime}=0
$$

where $A_{\mu}^{ \pm \prime}=\left(A_{\mu}^{4} \pm i A_{\mu}^{5}\right) / \sqrt{2}$. Finally, $U$-spin gauge transformations lead to

$$
\partial_{\mu} A_{\mu}^{ \pm \prime \prime} \pm i A_{\mu}^{3 \prime \prime} A_{\mu}^{ \pm \prime \prime}=0
$$

where $A_{\mu}^{ \pm \prime \prime}=\left(A_{\mu}^{6} \pm i A_{\mu}^{7}\right) / \sqrt{2}$. So the $S U(3)$ maximal abelian gauge functional Eq.(许) leads to the requirement that the gauge fields be 'maximally abelian' with respect to each of the three $S U(2)$ subgroups of $S U(3)$.

Minimizing the continuum functional Eq.(14) is equivalent to maximizing the lattice functional $\mathbf{G}_{\text {mag }}^{\text {su3 }}$ defined by

$$
\begin{aligned}
\mathbf{G}_{\text {mag }}^{\text {su3 }} & =\sum_{x, \mu}\left(\operatorname{tr}\left(U_{\mu}(x) \lambda_{3} U_{\mu}^{\dagger}(x) \lambda_{3}\right)+\operatorname{tr}\left(U_{\mu}(x) \lambda_{8} U_{\mu}^{\dagger}(x) \lambda_{8}\right)\right) \\
& =2 \sum_{x, \mu}\left(\left|\left(U_{\mu}\right)_{11}\right|^{2}+\left|\left(U_{\mu}\right)_{22}\right|^{2}+\left|\left(U_{\mu}\right)_{33}\right|^{2}-1\right) .
\end{aligned}
$$

We will use Eq.(8) for our MAG gauge-fixing in this paper. This same functional has been used in the small number of other papers published on the MAG for $S U(3)$ [5, 6].

At a local maximum of Eq.(\$), three conditions are satisfied. These are the lattice analogs of the continuum conditions Eqs.(3),(6), and (7), and are satisfied when $\mathbf{G}_{m a g}^{\text {su }}$ is stationary with respect to gauge transformations in the $I, U$, and $V$-spin subgroups. To write the conditions explicitly, we define three site-valued matrices. For the $I$-spin subgroup, the matrix $\mathbf{X}(x)$ is

$$
\mathbf{X}(x) \equiv \sum_{\mu}\left[U_{\mu}(x) \lambda_{3} U_{\mu}^{\dagger}(x)+U_{\mu}^{\dagger}(x-\hat{\mu}) \lambda_{3} U_{\mu}(x-\hat{\mu})\right] .
$$

For the $V$ and $U$-spin subgroups, the corresponding matrices $\mathbf{X}^{\prime}$ and $\mathbf{X}^{\prime \prime}$ are obtained by replacing $\lambda_{3}$ in Eq.(9) by $\lambda_{3}^{\prime}$ and $\lambda_{3}^{\prime \prime}$, respectively, where

$$
\lambda_{3}^{\prime} \equiv \frac{1}{2} \lambda_{3}+\frac{\sqrt{3}}{2} \lambda_{8}, \lambda_{3}^{\prime \prime} \equiv-\frac{1}{2} \lambda_{3}+\frac{\sqrt{3}}{2} \lambda_{8}
$$

When $\mathbf{G}_{\text {mag }}^{\text {su3 }}$ is stationary against gauge transformations in the $I$-spin subgroup, the 12 and 21 matrix elements of $\mathbf{X}(x)$ must vanish; similarly, for the $V$-spin subgroup, the 13 and 31 matrix elements of $\mathbf{X}^{\prime}(x)$ must vanish; and for the $U$-spin subgroup, the 23 and 32 matrix elements of $\mathbf{X}^{\prime \prime}(x)$ must vanish. In practice, we require that these matrix elements be very small. The numerical methods we use to reach these conditions with high accuracy will be discussed in $\operatorname{Sec}(5)$. 


\subsection{Generalized Maximal Abelian Gauge}

So far there appears to be a close analogy between the MAG conditions for $S U(2)$ and $S U(3)$. However, there are more general possibilities for $S U(3)$. To see them, we first note that $G_{m a g}^{s u 2}$ is in the form of a mass term for the gauge fields $A_{\mu}^{1}, A_{\mu}^{2}$. Such a term would arise in a gauge-Higgs theory, with an adjoint Higgs field $\Phi^{a}$ coupled to the $S U(2)$ gauge field. If the Higgs field has a vacuum expected value along the 3-axis, equal mass terms for $A_{\mu}^{1}$ and $A_{\mu}^{2}$ will be generated. Take the nondynamical Higgs field to have a fixed length, $\Phi^{a} \Phi^{a}=\Phi_{0}^{2}$, and write the continuum Higgs action

$$
G_{\text {higgs }}^{\text {su2 }}=\frac{1}{2} \int d^{4} x D_{\mu} \vec{\Phi} \cdot D_{\mu} \vec{\Phi}
$$

where $\left(D_{\mu} \Phi\right)^{a}=\partial_{\mu} \Phi^{a}+\epsilon^{a b c} A_{\mu}^{b} \Phi^{c}$. In the Higgs gauge with $\vec{\Phi}=\Phi_{0} \hat{3}, G_{\text {higgs }}^{\text {su2 becomes }}$

$$
G_{\text {higgs }}^{\text {su2 }}=\frac{\Phi_{0}^{2}}{2} \int\left(\left(A_{\mu}^{1}\right)^{2}+\left(A_{\mu}^{2}\right)^{2}\right) d^{4} x
$$

which is up to a constant, the same as $G_{m a g}^{s u 2}$.

The $S U(3)$ functional Eq. (团) is also in the form of mass terms for the charged gauge fields. It is of interest to see how the functional Eq.(4) is related to the action of a fixed length adjoint Higgs field $\vec{\Phi}$, with components $\Phi^{a}$. We write the continuum action

$$
G_{\text {higgs }}^{\text {su3 }}=\frac{1}{2} \int d^{4} x D_{\mu} \vec{\Phi} \cdot D_{\mu} \vec{\Phi}
$$

where $\left(D_{\mu} \Phi\right)^{a}=\partial_{\mu} \Phi^{a}+f^{a b c} A_{\mu}^{b} \Phi^{c}$. For $S U(3)$, going to a Higgs gauge means putting the Higgs field in the Cartan algebra, $\vec{\Phi}=\Phi_{0}(\hat{3} \cos \chi+\hat{8} \sin \chi)$, or in matrix form $\Phi=\Phi_{0}\left(\lambda_{3} \cos \chi+\lambda_{8} \sin \chi\right) / 2$. The value of the angle $\chi$ cannot be changed by an $S U(3)$ gauge transformation, so there are distinct 'moduli' or Higgs gauges parameterized by $\chi$. This is a difference between $S U(2)$ and $S U(3)$. In a generalized Higgs gauge where we allow $\chi$ to depend on $x$, the functional Eq.(13) takes the form

$$
\begin{aligned}
G_{\text {higgs }}^{\text {su } 3}= & \frac{1}{2} \Phi_{0}^{2} \int d^{4} x\left\{\partial_{\mu} \chi \partial_{\mu} \chi+\cos ^{2}(\chi)\left[\left(A_{\mu}^{1}\right)^{2}+\left(A_{\mu}^{2}\right)^{2}\right]+\right. \\
& \left.\cos ^{2}\left(\frac{\pi}{3}-\chi\right)\left[\left(A_{\mu}^{4}\right)^{2}+\left(A_{\mu}^{5}\right)^{2}\right]+\cos ^{2}\left(\frac{\pi}{3}+\chi\right)\left[\left(A_{\mu}^{6}\right)^{2}+\left(A_{\mu}^{7}\right)^{2}\right]\right\} .
\end{aligned}
$$

Even for $\chi$ independent of $x$, this is clearly different from Eq.(4). No individual adjoint Higgs field can give equal coefficients for the various charged vector fields in the functional. Formally, equal coefficients for the gauge fields can be obtained by taking $\chi$ to be independent of $x$, and then averaging over $\chi$ in Eq.(14).

Minimizing the functional Eq.(14) in the continuum is equivalent on the lattice to maximizing the functional $\mathbf{G}_{\text {higgs }}^{\text {su3 }}$ defined by

$$
\mathbf{G}_{\text {higgs }}^{\text {su3 }}=\sum_{x, \mu} \operatorname{tr}\left(\left(\lambda_{3} \cos \chi+\lambda_{8} \sin \chi\right) U_{\mu}(x)\left(\lambda_{3} \cos \chi^{\prime}+\lambda_{8} \sin \chi^{\prime}\right) U_{\mu}^{\dagger}(x)\right)
$$


where $\chi=\chi(x)$ and $\chi^{\prime}=\chi(x+\hat{\mu} a)$. If $\chi$ is taken to be independent of $x$, and $\mathbf{G}_{\text {higgs }}^{\text {su3 }}$ is averaged over $\chi, \mathbf{G}_{\text {higgs }}^{\text {su3 }}$ reduces to $\mathbf{G}_{\text {mag }}^{\text {su3 }}$. Although not used in the present paper, the functional $\mathbf{G}_{\text {higgs }}^{\text {su3 }}$ would appear to be an interesting alternative to $\mathbf{G}_{\text {mag }}^{\text {su3 }}$, particularly for the case where $\chi$ is allowed to depend on $x$. We intend to explore this in future work.

\subsection{Abelian Projection}

After the MAG gauge-fixing process is complete on a given configuration, each link $U_{\mu}$ is factored into a diagonal $U(1) \times U(1)$ part $u_{\mu}$, times a 'charged' part $U_{\mu}^{c h}$, so $U_{\mu}=u_{\mu} U_{\mu}^{c h}$. The $u_{\mu}$ has generators in the Cartan algebra and represents the 'phase' of the full $S U(3)$ link matrix $U_{\mu}$. Short-range physics is hopefully isolated in the matrix $U_{\mu}^{c h}$, which depends on the remaining six angles. In contrast to the $S U(2)$ case, simply extracting the phases of the diagonal elements of $U_{\mu}$ will not result in a matrix $u_{\mu}$ with $\operatorname{det}\left(u_{\mu}\right)=1$. In this paper, we use two different methods to extract $u_{\mu}$. In the 'symmetric' method, the phase of a given element of $u_{\mu}$ is set equal to the phase of the corresponding diagonal element of $U_{\mu}$, minus the average of the three diagonal phases. In the second, 'optimal' method, we find the $U(1) \times U(1)$ link which maximizes $\operatorname{Re}\left(\operatorname{tr}\left(u_{\mu} U_{\mu}^{\dagger}\right)\right.$ [7]. This finds the 'best' $U(1) \times U(1)$ approximation to $U_{\mu}$, and requires an iterative procedure. We have carefully checked that results produced by symmetric or optimal methods differ by a negligible amount, considerably smaller than statistical errors.

Once the $U(1) \times U(1)$ links $u_{\mu}$ are determined, the abelian projection is carried out by setting $U_{\mu}^{c h}$ to the identity on every link of the lattice, so the resulting $S U(3)$ links are equal to the $u_{\mu}$. Using the $u_{\mu}$ in place of the $U_{\mu}$ directly tests abelian dominance for $S U(3)$. The results of these calculations are reported in Sec.(5).

\section{$2.3 \mathrm{SU}(3)$ Monopoles}

In this section, we discuss the extraction of magnetic currents and calculations with monopoles for $S U(3)$, after reviewing the $S U(2)$ case [8]. There, after MAG gauge-fixing and abelian projection, the links are diagonal $2 \times 2$ matrices $u_{\mu}$ given by

$$
u_{\mu}=\left(\begin{array}{cc}
\exp \left(i \phi_{\mu}^{11}\right) & 0 \\
0 & \exp \left(i \phi_{\mu}^{22}\right)
\end{array}\right)
$$

where $\phi_{\mu}^{j j} \epsilon(-\pi,+\pi), j=1,2$ and $\phi_{\mu}^{11}+\phi_{\mu}^{22}=0$. Applying the Toussaint-DeGrand procedure [9] to plaquettes formed from the $\phi_{\mu}^{j j}$ gives two integer-valued magnetic currents $m_{\mu}^{j}$. However, $m_{\mu}^{1}+m_{\mu}^{2}=0$ is satisfied explicitly on every link, so there is only one magnetic current for $S U(2)$.

For $S U(3)$, after going to the MAG and projecting to $U(1) \times U(1)$, the abelian links are of the form

$$
u_{\mu}=\left(\begin{array}{ccc}
\exp \left(i \phi_{\mu}^{11}\right) & 0 & 0 \\
0 & \exp \left(i \phi_{\mu}^{22}\right) & 0 \\
0 & 0 & \exp \left(i \phi_{\mu}^{33}\right)
\end{array}\right)
$$


with $\phi_{\mu}^{11}+\phi_{\mu}^{22}+\phi_{\mu}^{33}=0, \bmod (2 \pi)$. Our procedure for extracting magnetic currents closely follows that just discussed for $S U(2)$. Plaquette angles are computed for each color, and the Toussaint-DeGrand procedure is applied to each. This produces a magnetic current for each color, $m_{\mu}^{j}, j=1,2,3$. Because of the $\bmod (2 \pi)$ in the sum of the angles $\phi_{\mu}^{j j}$, the total magnetic current $m_{\mu}^{1}+m_{\mu}^{2}+m_{\mu}^{3}$ does not vanish link by link. However, as shown in Sec.(6), the total magnetic current has no physical effect, so there are actually only two independent physical magnetic currents. This method of treating the magnetic current has the advantage that it does not force a particular subgroup structure for $S U(3)$ monopoles. Evidence that they are in fact associated with $S U(2)$ subgroups is presented elsewhere [10].

The monopole Wilson loops are calculated exactly as in $S U(2)$ or $U(1)$ [2]. Each color of magnetic current produces a lattice magnetic vector potential $A_{m \mu}^{j}$, and field strength $F_{m \mu \nu}^{j}=\partial_{\mu}^{+} A_{m \nu}^{j}-\partial_{\nu}^{+} A_{m \mu}^{j}$. The monopole Wilson loop is then obtained from the flux of the dual of $F_{m \mu \nu}^{j}$ through the loop. We have

$$
\left\langle W_{m o n}^{j}\right\rangle=\left\langle\exp \left(i 2 \pi \sum_{x, \mu>\nu} D_{\mu \nu}(x)^{*} F_{m \mu \nu}^{j}(x)\right)\right\rangle_{m},
$$

where $D_{\mu \nu}$ is unity on any simple surface of plaquettes whose edge is the Wilson loop, and $<>_{m}$ means the average over the ensemble of magnetic currents. The dual field strength is defined by ${ }^{*} F_{m \mu \nu}^{j}=\epsilon_{\mu \nu \alpha \beta} F_{m \alpha \beta}^{j} / 2$. Before extraction of potentials, the $\left\langle W_{m o n}^{j}>\right.$ are averaged over color.

\section{Maximal Center Gauge}

Gauge transformations involving a discrete subgroup are well-defined when acting on a path ordered exponential of gauge fields,

$$
U\left(x_{1}, x_{2}\right) \equiv P\left(\exp \left(\int_{x_{1}}^{x_{2}} A_{\mu} d x_{\mu}\right)\right) .
$$

Hence a gauge can be sought where a set of $U\left(x_{1}, x_{2}\right)$ are as close as possible to elements of a discrete subgroup of the gauge group.

In lattice gauge theory, the $U\left(x_{1}, x_{2}\right)$ are taken to be the links of the lattice. The direct maximal center gauge for $S U(N)$ lattice gauge theory seeks maxima of the functional

$$
\mathbf{G}_{d m c g}^{s u N}=\sum_{x, \mu}\left|\operatorname{tr} U_{\mu}\right|^{2}
$$

over the $S U(N)$ gauge group. Since $\operatorname{tr} U_{\mu}$ is real for $S U(2)$, an alternate form for $S U(2)$ is

$$
\mathbf{G}_{d m c g}^{s u 2}=\sum_{x, \mu}\left(\operatorname{tr} U_{\mu}\right)^{2}
$$


The $U_{\mu}$ are in the fundamental representation in all the formulae above. Fundamental and adjoint traces are related by $\operatorname{tr}\left(U_{\mu}^{a d j}\right)=\left|\operatorname{tr} U_{\mu}\right|^{2}-1$, so Eq.(19) can be rewritten as

$$
\mathbf{G}_{d m c g}^{s u N}=\sum_{x, \mu}\left(\operatorname{tr}\left(U_{\mu}^{a d j}\right)+1\right),
$$

which shows that the DMCG can be thought of as an adjoint Landau gauge. Gauge transformations are made so as to make the gauge-fields as small as possible, but any $Z(N)$ factors in the fundamental links are left unfixed.

Although we will restrict ourselves to the bilinear condition Eq.(19) in this work, it is worth noting that for $N>2$, there are additional $Z(N)$ invariant functionals of the links that can be constructed [11]. For example in $S U(3)$, the expressions $\operatorname{tr}\left(U_{\mu} U_{\mu} U_{\mu}\right)$, $\operatorname{tr}\left(U_{\mu} U_{\mu}\right) \cdot \operatorname{tr} U_{\mu}$, and $\operatorname{tr}\left(U_{\mu}\right) \cdot \operatorname{tr}\left(U_{\mu}\right) \cdot \operatorname{tr}\left(U_{\mu}\right)$ are all $Z(3)$ invariant. From these a family of real-valued, $Z(3)$ invariant functionals of the links could be written down and used to define $S U(3)$ maximal center gauges. It would be interesting to know if such gauges differ in their predictions from Eq.(19).

\subsection{Indirect Maximal Center Gauge}

In this paper, we take the indirect route to the maximal center gauge for $S U(3)$. This is the same method first used for $Z(2)$ center projection in $S U(2)$ [12], and fits naturally with our study of the MAG for $S U(3)$. A desirable feature of the IMCG is that it allows a study of the relationship between monopoles and center vortices. We start by going to the MAG as discussed in Sec.(2), using the functional of Eq.(8). The resulting $S U(3)$ links $U_{\mu}$ are then projected to $U(1) \times U(1)$ links $u_{\mu}$ using the methods discussed in Sec.(2.2). Parameterizing the $u_{\mu}$ as in Eq.(17), and using them to evaluate Eq.(19), the functional to be maximized for the IMCG becomes

$$
\begin{aligned}
& \mathbf{G}_{i m c g}^{\text {su3 }}=\sum_{x, \mu} {\left[3+2 \cos \left(\phi_{\mu}^{11}(x)-\phi_{\mu}^{22}(x)\right)+2 \cos \left(\phi_{\mu}^{11}(x)+2 \phi_{\mu}^{22}(x)\right)\right.} \\
&\left.+2 \cos \left(\phi_{\mu}^{22}(x)+2 \phi_{\mu}^{11}(x)\right)\right]
\end{aligned}
$$

where the unitarity condition has been used to eliminate $\phi_{\mu}^{33}$. The gauge functional of Eq.(22) is maximized by successively applying abelian gauge transformations at each site. The gauge transformations are parameterized as

$$
g(x)=e^{i \tilde{\lambda}_{8} \alpha_{8}(x)} e^{i \lambda_{3} \alpha_{3}(x)},
$$

where

$$
\tilde{\lambda_{8}} \equiv \operatorname{diag}(1,1,-2), \lambda_{3} \equiv \operatorname{diag}(1,-1,0) .
$$

Maximizing at a site $x$ gives two coupled equations in $\alpha_{3}(x)$ and $\alpha_{8}(x)$. These equations are not algebraically soluble, and their solution is found iteratively. Making the required gauge transformation at $x$ generally causes the maximization conditions at the nearest neighbors of $x$ to be violated, so reaching a local maximum of $\mathbf{G}_{i m c g}^{\text {su3 }}$ requires repeated 
cycles through the lattice. At a maximum, two gauge conditions are satisfied. The first comes from the $\lambda_{8}$ term in the $U(1) \times U(1)$ gauge transformation,

$$
\sum_{\mu} \partial_{\mu}^{-}\left[\sin \left(2 \phi_{\mu}^{11}(x)+\phi_{\mu}^{22}(x)\right)+\sin \left(2 \phi_{\mu}^{22}(x)+\phi_{\mu}^{11}(x)\right)\right]=0 .
$$

The second comes from the $\lambda_{3}$ term,

$$
\sum_{\mu} \partial_{\mu}^{-}\left[2 \sin \left(\phi_{\mu}^{11}(x)-\phi_{\mu}^{22}(x)\right)+\sin \left(2 \phi_{\mu}^{11}(x)+\phi_{\mu}^{22}(x)\right)-\sin \left(2 \phi_{\mu}^{22}(x)+\phi_{\mu}^{11}(x)\right)\right]=0 .
$$

In the naive continuum limit these two conditions reduce to the abelian Landau gauge conditions

$$
\partial_{\mu} A_{\mu}^{8}=0, \partial_{\mu} A_{\mu}^{3}=0
$$

where $a A_{\mu}^{8}=\left(\phi_{\mu}^{11}(x)+\phi_{\mu}^{22}(x)\right) / 2$, and $a A_{\mu}^{3}=\left(\phi_{\mu}^{11}(x)-\phi_{\mu}^{22}(x)\right) / 2$. In our lattice calculations, the right hand sides of Eqs.(23) and (24) are not actually zero, but are very small. This is discussed in Sec.(7).

After a local maximum is reached to sufficient accuracy, the links $u_{\mu}$ are projected to the nearest element of the center group, $Z(3)$, where

$$
Z(3) \in\{\exp (-i 2 \pi / 3), 1, \exp (+i 2 \pi / 3)\} \times \mathbf{1} .
$$

This results in a set of $Z(3)$-valued links, $z_{\mu}(x)$. These are used to compute the $Z(3)$ Wilson loops discussed in Sec.(17).

Since our results for center projection are all for the IMCG, it is worth commenting on possible differences between the direct and indirect maximal center gauges. The IMCG depends on the specific functional form used for the MAG functional, which as discussed in Sec.(2), is not unique for $S U(3)$. This means the IMCG results obtained in this paper using the Eq.(8) form of the MAG are not necessarily indicative of those that would be obtained in the DMCG for $S U(3)$. For the case of an $S U(2)$ gauge group, where the MAG functional is unambiguous, we have found no statistically significant difference between indirect and direct methods of reaching the maximal center gauge[13]. A similar result may hold in the end for $S U(3)$, but this will probably require an MAG functional different from Eq.(8).

\section{$4 \quad S U(3)$ Results}

Our calculations were performed at couplings $\beta=5.9$ and 6.0 on lattices of size $10^{3} \times 16$ and $16^{4}$, respectively. The correlation length as determined by the string tension is approximately $4 a$ at $\beta=5.90$ and $4.5 a$ at $\beta=6.0$, so at $\beta=5.90$, our $10^{3} \times 16$ lattice is over twice the correlation length in size, while at $\beta=6.0$, the $16^{4}$ lattice is more than three times as large as the correlation length. Finite size effects should be small, particularly at $\beta=6.0$. In each case, $S U(3)$ configurations were generated using the Wilson action with a heatbath update and overrelaxation [14]. The first several thousand configurations were 
discarded to allow for equilibration, and every $20^{\text {th }}$ configuration after that was used for measurements. We analyzed 440 configurations at $\beta=5.9$, and 600 at $\beta=6.0$.

In this section, we present full $S U(3)$ results for the heavy quark potential. Results for the MAG $U(1) \times U(1)$, MAG monopole and IMCG $Z(3)$ potentials are presented in the sections immediately following. The full $S U(3)$ potential is of course gauge-invariant, and therefore not subject to the uncertainties of gauge-fixing and projection. On the other hand, the noise level of $S U(3)$ Wilson loops is much greater than for projected loops, and variance reduction is essential to extract the potential. The presence of a large Coulomb term also complicates the determination of the string tension. For the $S U(3)$ potential, modified Wilson loops were computed using a multihit variance reduction scheme [15] and spatial smearing [16]. In computing the heavy quark potential, we used loops of spatial extent $R=2 a$ to $R=5 a(\beta=5.9), R=2 a$ to $R=7 a(\beta=6.0)$. The maximum temporal extent was $T=12 a$ in both cases. From the Wilson loops, we extracted potentials by fitting $-\ln (W(R, T))$ to a straight line in $T$, the slope giving $V(R)$ [17. The values of $T$ included in the fits for each $R$ were $T=R+a$ to $T=12 a$. The potentials were then fitted to the form $V(R)=V_{0}+\alpha / R+\sigma R$.

Our results for the full $S U(3)$ potential parameters are presented in Table 1 . For the purposes of this paper, the string tensions in Table 1 are the most significant, since the various projection schemes we will compare to in later sections do not (nor are they expected to) reproduce the Coulomb part of the potential. Comparing our $S U(3)$ results to the modern high statistics calculations available in the literature, our $\beta=6.0$ string tension is within error bars of the values quoted in 18, 19, 20, 21. At $\beta=5.90$, the only calculation with comparable accuracy is [22], where the value reported is somewhat higher than ours, by an amount slightly outside of error bars. However, it should be noted that at $\beta=6.0$, the string tension quoted in [22] is also somewhat higher than the results in [18, 19, 20, 21]. We conclude that our full $S U(3)$ string tensions are of good quality, comparable to the best values available in the literature.

\section{$5 \quad U(1) \times U(1)$ Results}

This section contains our most important results. Abelian projection after gauge-fixing to the MAG retains the largest number of degrees of freedom of the various projections we consider, and so is a less severe truncation of the theory than retaining only the abelian fields of monopoles, or keeping only $Z(3)$-valued links after center projection.

The process of actually finding a local maximum of the MAG functional was carried out iteratively, determining the gauge transformation $g(x)$ at a specific site which

maximizes the terms in $\mathbf{G}_{m a g}^{\text {su3 }}$ that are affected by $g(x)$. Gauge transformations were successively chosen from the three independent subgroups of $S U(3)$. An arbitrary $S U(3)$ gauge transformation can be expressed as a product of such $S U(2)$ gauge transformations. To improve convergence, we used an overrelaxation procedure, replacing $g(x)$ with $g^{\omega}(x)$ [23]. For gauge transformations which are elements of $S U(2)$ subgroups, it is possible to work out $g^{\omega}(x)$ exactly, rather than use the Taylor expansion approximation described in 
[23]. It was found that $\omega=1.85$ was the optimum overrelaxation parameter when using the exact expression for $g^{\omega}(x)$. Referring back to the conditions discussed after Eq.(8), gauge fixing iterations were performed until

$$
\left\langle\left|\mathbf{X}(x)_{12}\right|^{2}+\left|\mathbf{X}(x)_{21}\right|^{2}+\left|\mathbf{X}^{\prime}(x)_{13}\right|^{2}+\left|\mathbf{X}^{\prime}(x)_{31}\right|^{2}+\left|\mathbf{X}^{\prime \prime}(x)_{23}\right|^{2}+\left|\mathbf{X}^{\prime \prime}(x)_{32}\right|^{2}\right\rangle \leq 10^{-12},
$$

where $<>$ implies a lattice average. Eq.(25) is a very tight constraint. We are confident that when it is satisfied, the configuration is very close to a local maximum of $\mathbf{G}_{m a g}^{\text {su3 }}$. As with any maximal gauge, the functional $\mathbf{G}_{m a g}^{\text {su3 }}$ has many local maxima or gauge copies. A single gauge-fixing brings a configuration near a random local maximum of the functional, unrelated in general to the global maximum. Actually finding the global maximum is impractical. However, it is feasible to generate a sample of gauge copies for each configuration, and study how the projected string tension varies when copies with steadily increasing values of the functional are used. Our results are presented first for the case of one gauge copy/configuration, followed by analysis of the effect of gauge ambiguities.

After reaching a local maximum for each configuration, the $S U(3)$ links were projected to the $U(1) \times U(1)$ links $u_{\mu}$, using the methods discussed in Sec.(2.2). Spatial links were smeared using the Ape method [16] with a staple weighting parameter of 0.7 , iterating the procedure 8 times per link. Then abelian Wilson loops were computed using

$$
W_{a b e l}(R, T)=\left\langle\frac{1}{3}\left(\operatorname{tr} \prod_{C} u_{\mu}(x)\right)\right\rangle .
$$

These loops were used to compute the $U(1) \times U(1)$ heavy quark potential $V(R)$ at both $\beta=5.90$ and $\beta=6.0$. The results for fits to $V(R)$ of the same type as for the full $S U(3)$ potential are shown in Table 2 .

The $U(1) \times U(1)$ string tensions shown in Table 2 are for one local maximum or gauge copy/configuration, and are smaller than the corresponding full $S U(3)$ results, by $10 \%$ at $\beta=6.0$. This is in contrast to the behavior of $S U(2)$ in the MAG for one gauge copy/configuration. There similar gauge-fixing methods produce $U(1)$ string tensions at least $10 \%$ larger than full $S U(2)$ at $\beta_{S U(2)}=2.4,2.5,2.6$ [3, 24].

Turning to gauge ambiguities, in order for their effect to bring the $U(1) \times U(1)$ string tension closer to the full $S U(3)$ answer, local maxima of the gauge functional with higher values of the functional would have to produce higher abelian projected string tensions. This is the opposite of what happens in $S U(2)$. To explore whether this unexpected behavior occurs for the $S U(3)$ MAG functional of Eq.(8), we generated $N_{\text {copy }}=10$ gauge copies/configuration for each of 200 configurations at $\beta=6.0$. To generate a gauge copy, a move is made to an arbitrary point on the gauge orbit of a configuration by making a random gauge transformation. The gauge-fixing algorithm then takes the configuration to a nearby local maximum. Due to abelian projection, the $U(1) \times U(1)$ string tension is dependent on which copies are used for each configuration. Our treatment of the variation due to gauge copies follows the method of Bali, et. al. [24, developed for the $S U(2)$ case. For each configuration, a subset of size $n_{g}$ of the $N_{\text {copy }}$ copies is chosen. Only the configuration in the subset with the highest value of the gauge functional is retained 
and used to calculate Wilson loops. The Wilson loops are averaged over all such subsets of size $n_{g}$. For $n_{g}=1$, this is just the average over all $N_{\text {copy }}$ copies for each configuration, whereas for $n_{g}=N_{\text {copy }}$, only the copy with the highest value of the functional for all $N_{\text {copy }}$ copies is used for each configuration. The resulting Wilson loops are then averaged over configurations and used to calculate potentials. The output of this analysis is a set of values of the $U(1) \times U(1)$ string tension for $n_{g}$ ranging from 1 to $N_{\text {copy }}$. The results are shown in Table 3. As can be seen there, the $U(1) \times U(1)$ string tension steadily decreases as $n_{g}$ increases. At $n_{g}=N_{\text {copy }}=10$, the $U(1) \times U(1)$ string tension has decreased by $10 \%$, and the ratio of the $U(1) \times U(1)$ to the $S U(3)$ string tension is $0.80(4)$.

We have thus found that the effect of gauge ambiguities in $S U(3)$ is similar to $S U(2)$, namely, the higher the value of the MAG functional, the lower the abelian projected string tension. In $S U(2)$, this trend is beneficial, since for a randomly chosen gauge copy, the $U(1)$ string tension is above the full $S U(2)$ result, and taking gauge copies with successively higher values of the functional brings the string tension down, closer and closer to the full $S U(2)$ value. For $S U(3)$, using the Eq.(8) form of the MAG, the $U(1) \times U(1)$ string tension from a random gauge copy is too low to start with, and gauge copies with higher functional values produce $U(1) \times U(1)$ string tensions still further below the $S U(3)$ string tension.

\section{Monopole Results}

Before presenting results on the heavy quark potential due to monopoles, we discuss a point mentioned in Sec.(2.3), related to the determination of the magnetic current. Our procedure for locating monopoles produces a separate current $m_{\mu}^{j}$ for each color, $j=1,2,3$. This method treats the three colors symmetrically, and is not prejudiced toward a specific group structure for the monopoles. Only two of these currents are really independent. Although it does not vanish link by link, the total current, $m_{\mu}^{1}+m_{\mu}^{2}+m_{\mu}^{3}$, is in effect a null current. We have checked this by using the total current to calculate monopole Wilson loops. The resulting string tension (at $\beta=5.90$ ) is zero to four decimal places, $\sigma_{m o n}^{\text {tot }}=0.0000(4)$, as it should be.

Turning to the monopole potentials, at $\beta=5.9$ and 6.0 , we computed monopole Wilson loops for each color using Eq.(18), averaged the results over color, and extracted heavy quark potentials using the same fit methods discussed earlier. The results are presented in Table 1 along with the current density, or fraction $f_{m}$ of links carrying magnetic current. The results shown in Table 1 are for one MAG gauge-fixing/configuration, and are on the same footing as the $U(1) \times U(1)$ results in Table 2 . The inequality $\sigma_{S U(3)}>\sigma_{U(1) \times U(1)}>\sigma_{\text {mono }}$ is seen to hold for both $\beta$ values, the monopole string tensions being approximately $25 \%$ smaller than the $S U(3)$ string tensions, a discrepancy well outside of error bars.

It is of interest to compare these results with corresponding ones for $S U(2)$. The full $S U(2)$ string tensions at $\beta_{S U(2)}=2.4$ and $\beta_{S U(2)}=2.45$ are numerically close to the full $S U(3)$ string tensions at $\beta_{S U(3)}=5.9$ and $\beta_{S U(3)}=6.0$, respectively, implying similar 
correlation lengths. However, for $S U(2)$, the monopole string tensions for one gaugefixing/configuration are within $5 \%$ of the full $S U(2)$ values [8]. Further, the magnetic current densities $f_{m}$, are more than twice as large as the corresponding $S U(3)$ results shown in Table 1 . Thus for $S U(3)$, the MAG condition of Eq.(8) leads to projected configurations with a severe shortage of monopoles. Despite this shortage, the magnetic current density shows scaling properties rather similar to those found in $S U(2)$. If the magnetic current density is a physical density, dividing it by the cube of a physical energy should produce a scaling quantity, i.e. one independent of $\beta$. Computing $f_{m} /\left(\sigma_{m o n o}\right)^{3 / 2}$ for $\beta=5.90$ and $\beta=6.0$ gives 1.1(1) and 1.0(1), respectively. Corresponding results for $S U(2)$ at $\beta_{S U(2}=2.40$ and $\beta_{S U(2}=2.45$ are $1.6(1)$ and $1.7(1)$, respectively [8]. So although the magnetic current density for $S U(3)$ is significantly smaller, it scales with $\beta$ in a similar way to the $S U(2)$ case when the monopole string tension defines the unit of energy.

Just as they affect the $U(1) \times U(1)$ potential, MAG gauge ambiguities affect the monopole potential parameters. Using the same set of $N_{\text {copy }}=10$ gauge copies/configuration at $\beta=6.0$, we can determine how the monopole string tension and magnetic current density vary with the number of gauge copies. The analysis proceeds exactly as in the $U(1) \times U(1)$ case. The results are presented in Table . . As $n_{g}$ increases, the monopole string tension decreases by essentially the same factor as the $U(1) \times U(1)$ string tension, and the ratio of the magnetic current density to $\left(\sigma_{m o n}\right)^{3 / 2}$ remains approximately constant. By the time $n_{g}=N_{\text {copy }}=10$ has been reached, the $\beta=6.0$ value of the monopole string tension is $34 \%$ smaller than the $S U(3)$ string tension.

\section{$7 \quad Z(3)$ Results}

To transform an $S U(3)$ configuration to the indirect maximal center gauge requires two gauge-fixings and one projection. First a local maximum of the MAG functional is found, followed by abelian projection. The resulting $U(1) \times U(1) u_{\mu}$ links are then gauge-fixed by finding a local maximum of the IMCG functional Eq.(22), using the procedure described in Sec.(3.1). Iterations were performed until the sum of the squares of Eqs.(23) and (24) was less than $10^{-14}$, when averaged over the lattice. During the gauge fixing we monitored the modulus of the abelian trace, $\left|\operatorname{tr} u_{\mu}\right|$. If all links were elements of the center, the value of $\frac{1}{3}\left\langle\left|\operatorname{tr} u_{\mu}\right|\right\rangle$ would be unity. At $\beta=5.90$, we observed it to change from an average value of 0.525 before gauge fixing to a value of 0.922 after gauge fixing. The behavior at $\beta=6.0$ is similar.

The IMCG has gauge ambiguities of two types; those inherent in the MAG, as well as those that are associated with the second gauge-fixing just described. We first present results for one gauge-fixing of each type/configuration. This puts each configuration in a random local maximum of both gauge conditions. The resulting center-projected links were used to compute $Z(3)$ Wilson loops and extract a heavy quark potential as in the previous cases. Similar to the monopole case, the noise level is very low, and the potential is almost perfectly linear. Table 6 shows the potential parameters, along with $p$, 
the fraction of plaquettes with non-trivial $Z(3)$ flux. These plaquettes are conjectured to represent the geometrical centers of finite-sized center vortices. Usually called P-vortices, we simply refer to non-trivial $Z(3)$ plaquettes as vortices. From Table 6 and Table 1 , the ratio of the $Z(3)$ string tension to the full $S U(3)$ value is $0.88(8)$ at $\beta=5.9$, but falls to $0.80(8)$ at $\beta=6.0$.

The vortex density is an areal density, so if it is physical, dividing by the square of a physical energy should produce a scaling quantity. The natural quantity to divide by is the $Z(3)$ string tension. Computing the ratio of the vortex density to the $Z(3)$ string tension gives .73(1) and .75(1) for $\beta=5.90$ and $\beta=6.0$ respectively. For the DMCG in $S U(2)$ the results at $\beta_{S U(2}=2.40$ and $\beta_{S U(2}=2.50$ are quite similar; 0.76(1) and $0.78(1)$, respectively [13]. Thus for both $S U(3)$ and $S U(2)$, dividing the vortex density by the $Z(N)$ string tension removes most of the $\beta$-dependence and produces an approximate scaling quantity.

We also explored the effect of gauge ambiguities on the IMCG results. To avoid an expensive simultaneous treatment of both MAG and IMCG gauge ambiguities, we generated 10 IMCG gauge copies only for the MAG copy with the highest value of the MAG functional. This is the best MAG copy from the viewpoint of the MAG functional. The analysis of the $Z(3)$ string tension proceeded in exactly the same manner as the $U(1) \times U(1)$ case. The results are shown in Table 0, where it is seen that the $Z(3)$ string tension decreases by $\mathrm{O}(10 \%)$ in going from $n_{g}=2$ to $n_{g}=10$. The ratio of vortex density to $Z(3)$ string tension is approximately constant. At $n_{g}=10$, value of the $Z(3)$ string tension is roughly $40 \%$ smaller than the $\beta=6.0 S U(3)$ string tension.

If a different choice of MAG copy had been made, it is reasonable to expect a similar $\mathrm{O}(10 \%)$ variation due to IMCG gauge ambiguities. If for example, a random MAG gauge copy is used for each configuration and IMCG copies generated, the $Z(3)$ string tension would be expected to decrease from its value given in Table 6 to one $\mathrm{O}(10 \%)$ lower, so for $n_{g}=10$, the $Z(3)$ string tension would be $\mathrm{O}(70 \%)$ of the $\beta=6.0 S U(3)$ string tension.

A reasonable conclusion is that using the gauge functionals Eq.(8) for the MAG and Eq.(22) for the IMCG, whatever choice is made of MAG or IMCG gauge copies, the result is a $\beta=6.0 Z(3)$ string tension whose central value is at least $20 \%$ smaller than the full $S U(3)$ string tension.

Finally, it is of interest to study the connection between vortices and monopoles. Suppose the magnetic current is non-zero on a dual lattice link. This link is dual to a 3 -cube on the original lattice. The partial magnetic current density $f_{m}(n)$ is defined as the current density for links where the dual cube has $n$ faces pierced by $Z(3)$ vortices. The total magnetic current density is then

$$
f_{m}=\sum_{n=0}^{6} f_{m}(n) .
$$

Since $Z(3)$ center vortices form closed surfaces, $f_{m}(1) \equiv 0$, but all other possibilities can and do occur. The study was carried out for one gauge-fixing of each type/configuration. We computed the ratio $r_{m}(n)=f_{m}(n) / f_{m}$, which measures the fractional occurrence for 
the cube dual to a magnetic current link to have $n$ faces pierced by $Z(3)$ vortices. The results are shown in Table 8 , and hold independent of color. Thus approximately 85\% $(83 \%)$ of the time at $\beta=5.9(6.0)$, the cube dual to a magnetic current link has at least two faces pierced by vortices. This says there is an intimate connection between center vortices and monopoles in $S U(3)$, similar to what was found previously in $S U(2)$ 13, 25. It could in fact be true that the actual fractions are $100 \%$, with the smaller numerical results caused by inaccuracies in locating monopoles and vortices on the lattice.

\section{Summary and Conclusions}

We have calculated the heavy quark potential for $S U(3)$ lattice gauge theory at $\beta=5.90$ and $\beta=6.0$. These $S U(3)$ results have been compared with three cases which project onto a reduced set of degrees of freedom after gauge-fixing. The $U(1) \times U(1)$ and monopole results involve the version of the MAG expressed in Eq.(8), while the $Z$ (3) results involve in addition the IMCG gauge-fixing of Eq.(22). In Figs.(1) and (2), we show plots of the full $S U(3)$ and projected potentials, for the case of one gauge-fixing/configuration. The potentials have been shifted by constants for convenient display, the significant quantity to compare being the slope of each potential at large $\mathrm{R}$, which determines the string tension. The string tension results obey the inequalities $\sigma_{S U(3)}>\sigma_{U(1) \times U(1)}>\sigma_{Z(3)}>\sigma_{m o n o}$. These inequalities hold at one gauge-fixing/configuration and become stronger when account is taken of gauge ambiguities. The most important result of this paper is that $\sigma_{S U(3)}>\sigma_{U(1) \times U(1)}$. When account is taken of gauge ambiguities at $\beta=6.0$, the $U(1) \times U(1)$ string tension is smaller than the full $S U(3)$ string tension by $20 \%$, an amount well outside of error estimates. If a simulated annealing gauge-fixing algorithm [3, 24] were used instead of the overrelaxation method used here, the discrepancy could be still larger. This situation for $S U(3)$ is in contrast to $S U(2)$, where the MAG and extrapolating to the global maximum of the functional $\left(n_{g} \rightarrow \infty\right)$ produces a $U(1)$ string tension very close to the full $S U(2)$ result [3].

It is conceivable that for $S U(3)$, the discrepancy between $\sigma_{S U(3)}$ and $\sigma_{U(1) \times U(1)}$ could fade away with increasing $\beta$ and become negligible in the continuum limit. This seems unlikely given that the ratio $\sigma_{U(1) \times U(1)} / \sigma_{S U(3)}$ is smaller at $\beta=6.0$ than $\beta=5.9$. Rather than moving into the region $\beta>6.0$ using the Eq.(8) form of the MAG, we intend to explore the possibility that the remedy lies in a generalized MAG such as Eq.(15). The condition of Eq.(15) seems to express in a natural way the idea that there is an effective adjoint Higgs field present, whose direction in the Cartan algebra may vary from point to point on the lattice. If it is accepted that the simple MAG form of Eq.(8) cannot work for $S U(3)$, it is important to explore more general forms of the MAG. Failure to find any form of MAG which works would have serious implications for 't Hooft's proposal of abelian dominance of confinement for $S U(N), N>2$. On the other hand, a quantitatively successful generalized MAG in $S U(3)$ opens the possibility of a similar result for the indirect maximal center gauge, and a detailed study of the contribution to confinement of MAG monopoles and IMCG center vortices. These two degrees of freedom are clearly 
intimately connected.

With any generalized MAG, there will be a rather clear signal for improvement. An improved form of the MAG should produce a string tension larger than the full $S U(3)$ result for one gauge-fixing/configuration. Gauge copies with higher values of the functional will inevitably lower the abelian $U(1) \times U(1)$ string tension, hopefully to within error bars of the full $S U(3)$ result. In contrast, a form of the MAG which for one gaugefixing/configuration produces an abelian projected string tension smaller than the full $S U(3)$ result is untenable. Gauge copies with higher values of the functional will only reduce it further. Our claim is that the MAG in the form of Eq.(8) is of this type.

With regard to center projection, since $Z(3)$ is a subgroup of $U(1) \times U(1)$, the group center should be accessible indirectly, i.e. after abelian projection. However, given the subtleties of the MAG for $S U(3)$, a thorough study of the direct maximal center gauge for $S U(3)$ is quite desirable. The DMCG avoids the question of what is the proper form of the MAG. We intend to study the direct maximal center gauge for $S U(3)$ in future work.

\section{Acknowledgements}

This work was supported in part by the National Science Foundation under grants NSF PHY 9900658 (JDS) and NSF PHY 9802579 (RJW). The numerical computations were supported in part by NSF cooperative agreement ACI-9619020 through computing resources provided by the National Partnership for Advanced Computational Infrastructure at the San Diego Supercomputer Center and the Texas Advanced Computing Center.

\section{References}

[1] G. 't Hooft, "Topology of The Gauge Condition and Confinement Phases in Nonabelian Gauge Theories," Nucl. Phys. B190 (1981) 455.

[2] J. Stack and R. Wensley, "Monopoles, Quark Confinement and Screening in FourDimensional U(1) Lattice Gauge Theory," Nucl. Phys. B371 (1992) 597.

[3] V. Bornyakov and M. Müller-Preussker, "Continuum Limit in Abelian Projected SU(2) Lattice Gauge Theory," Nucl. Phys. B Proc. Suppl. 106 (2002) 646.

[4] V. G. Bornyakov, D. A. Komarov, and M. I. Polikarpov, "P Vortices and Drama of Gribov Copies," Phys. Lett. B497 (2001) 151.

[5] V. Bornyakov, H. Ichie, S. Kitahara, Y. Koma, Y. Nakamura, M. Polikparov, G. Schierholz, T. Streuer, H. Stüben, and T. Suzuki, "On the Dynamics of Color Magnetic Monopoles in Full QCD," hep-lat/0111042 (2001).

[6] F. Brandstater, G. Schierholz, and U.-J. Wiese, "Color Confinement, Abelian Dominance, and the Dynamics Of Magnetic Monopoles in SU(3) Gauge Theory," Phys. Lett. B272 (1991) 319. 
[7] W. W. Tucker and J. D. Stack, "The Maximal Abelian Gauge in SU(3) Lattice Gauge Theory," Nucl. Phys. B Proc. Suppl. 106 (2002) 643.

[8] J. D. Stack, S. D. Neiman, and R. J. Wensley, "String Tension from Monopoles in SU(2) Lattice Gauge Theory," Phys. Rev. D50 (1994) 3399.

[9] T. A. DeGrand and D. Toussaint, "Topological Excitation and Monte Carlo Simulation of Abelian Gauge Theory," Phys. Rev. D22 (1980) 2478.

[10] J. D. Stack, W. W. Tucker, and R. J. Wensley, "Confinement in SU(3): Simple and Generalized Maximal Abelian Gauge," hep-lat/0205006 (2002).

[11] M. Faber, J. Greensite, and S. Olejnik, "First Evidence for Center Dominance in SU(3) Lattice Gauge Theory," Phys. Lett. B474 (2000) 177.

[12] L. Del Debbio, M. Faber, J. Greensite, and Š. Olejník, "Some Cautionary Remarks on Abelian Projection and Abelian Dominance," Nuc. Phys. B(Proc. Suppl.) 53 (1997) 141.

[13] J. D. Stack, W. W. Tucker and A. Hart, "Cooling, Monopoles, and Vortices in SU(2) Lattice Gauge Theory," hep-lat/0011057 (2000).

[14] A. D. Kennedy and B. J. Pendelton, "Improved Heat Bath Method for Monte Carlo Calculations in Lattice Gauge Theory," Phys. Lett. B156 (1985) 393.

[15] G.Parisi, R. Petronzio, and R. Rapuano, "A Measurement of the String Tension near the Continuum Limit," Phys. Lett. B128 (1983) 418.

[16] The APE Collaboration(M. Albanese et. al), "Glueball Masses and String Tension in Lattice QCD," Phys. Lett.192B (1987) 163.

[17] J. D. Stack, "The Heavy Quark Potential in SU(2) Lattice Gauge Theory," Phys. Rev. D27 (1983) 412.

[18] S. Perantonis and C. Michael, "Static Potentials and Hybrid Mesons from Pure SU(3) Lattice Gauge Theory," Nucl. Phys. B347 (1990) 854.

[19] G.S. Bali and K. Schilling, "Static Quark-Antiquark Potential: Scaling Behavior and Finite Size Effects in SU(3) Lattice Gauge Theory," Phys. Rev. D 46 (1992) 2636.

[20] G.S. Bali and K. Schilling, "Running Coupling and the $\Lambda$ Parameter from SU(3) Lattice Simulations," Phys. Rev. D 47 (1993) 661.

[21] G.S. Bali, K. Schilling, and A. Wachter, "Complete $O\left(v^{2}\right)$ Corrections to the Static Interquark Potential from SU(3) Gauge Theory," Phys. Rev. D 56 (1997) 2566.

[22] K.D. Born, R. Altmeyer, W. Ibes, E. Laermann, R. Sommer, T.F. Walsh, and P.M. Zerwas, "The Interquark Potential," Nucl. Phys B (Proc. Suppl.) 20 (1991) 394. 
[23] J. Mandula and M. Ogilvie, "Efficient Gauge-fixing via Overrelaxation," Phys. Lett. B 248 (1990) 156.

[24] G. S. Bali, V. Bornyakov, M. Müller-Preussker, and K. Schilling, "Dual Superconductor Scenario of Confinement: A Systematic Study of Gribov Copy Effects," Phys. Rev. D54 (1996) 2863.

[25] L. Del Debbio, M. Faber, J. Greensite, and S. Olejnik, "Center Dominance, Center Vortices, and Confinement," hep-lat/9708023 (1997).

Table 1: Full $S U(3)$ results for string tension, Coulomb coefficient, and self energy at $\beta=5.90$ and $\beta=6.0$

\begin{tabular}{||c|c|c||}
\hline$S U(3)$ & $\beta=5.90$ & $\beta=6.0$ \\
\hline Vol & $10^{3} \times 16$ & $16^{4}$ \\
\hline$\sigma$ & $0.068(3)$ & $0.050(1)$ \\
\hline$\alpha$ & $-0.36(2)$ & $-0.32(2)$ \\
\hline$V_{0}$ & $0.68(2)$ & $0.66(2)$ \\
\hline $\mathrm{R} / \mathrm{a}$ & 2 to 5 & 2 to 7 \\
\hline
\end{tabular}

Table 2: MAG $U(1) \times U(1)$ results for string tension, Coulomb coefficient, and self energy at $\beta=5.90$ and $\beta=6.0$, one gauge copy/config.

\begin{tabular}{||c|c|c||}
\hline$U(1) \times U(1)$ & $\beta=5.90$ & $\beta=6.0$ \\
\hline Vol & $10^{3} \times 16$ & $16^{4}$ \\
\hline$\sigma$ & $0.063(3)$ & $0.045(2)$ \\
\hline$\alpha$ & $-0.09(2)$ & $-0.07(2)$ \\
\hline$V_{0}$ & $0.20(2)$ & $0.19(2)$ \\
\hline $\mathrm{R} / \mathrm{a}$ & 2 to 5 & 2 to 7 \\
\hline
\end{tabular}


Table 3: $\beta=6.0$ MAG $U(1) \times U(1)$ string tensions for subsets of size $n_{g}$ out of 10 gauge copies

\begin{tabular}{||c|c||}
\hline$n_{g}$ & $\sigma_{U(1) \times U(1)}$ \\
\hline 2 & $0.042(1)$ \\
\hline 4 & $0.041(1)$ \\
\hline 6 & $0.041(1)$ \\
\hline 8 & $0.040(1)$ \\
\hline 10 & $0.040(1)$ \\
\hline
\end{tabular}

Table 4: MAG monopole results for string tension, Coulomb coefficient, self energy, and current density, at $\beta=5.90$ and $\beta=6.0$, one gauge copy/configuration

\begin{tabular}{||c|c|c||}
\hline mono & $\beta=5.90$ & $\beta=6.0$ \\
\hline Vol & $10^{3} \times 16$ & $16^{4}$ \\
\hline$\sigma$ & $0.050(2)$ & $0.038(1)$ \\
\hline$\alpha$ & $0.03(2)$ & $0.045(1)$ \\
\hline$V_{0}$ & $-0.04(2)$ & $-0.051(4)$ \\
\hline$f_{m}$ & $1.28(1) \times 10^{-2}$ & $7.45(3) \times 10^{-3}$ \\
\hline $\mathrm{R} / \mathrm{a}$ & 2 to 6 & 2 to 8 \\
\hline
\end{tabular}

Table 5: $\beta=6.0$ MAG monopole string tensions and current densities for subsets of size $n_{g}$ out of 10 gauge copies

\begin{tabular}{||c|c|c||}
\hline$n_{g}$ & $\sigma_{\text {mono }}$ & $f_{m}$ \\
\hline 2 & $0.036(1)$ & $7.25(4) \times 10^{-3}$ \\
\hline 4 & $0.035(1)$ & $7.08(4) \times 10^{-3}$ \\
\hline 6 & $0.034(1)$ & $7.00(4) \times 10^{-3}$ \\
\hline 8 & $0.034(1)$ & $6.95(4) \times 10^{-3}$ \\
\hline 10 & $0.033(1)$ & $6.92(5) \times 10^{-3}$ \\
\hline
\end{tabular}


Table 6: IMCG results for $Z(3)$ string tension, Coulomb coefficient, self energy, and vortex density, at $\beta=5.90$ and $\beta=6.0$, one gauge copy/configuration

\begin{tabular}{||c|c|c||}
\hline$I M C G$ & $\beta=5.9$ & $\beta=6.0$ \\
\hline Vol & $10^{3} \times 16$ & $16^{4}$ \\
\hline$\sigma$ & $0.060(3)$ & $0.040(2)$ \\
\hline$\alpha$ & $0.05(2)$ & $0.03(1)$ \\
\hline$V_{0}$ & $-0.04(2)$ & $-0.02(1)$ \\
\hline$p$ & $4.40(1) \times 10^{-2}$ & $3.01(1) \times 10^{-2}$ \\
\hline $\mathrm{R} / \mathrm{a}$ & 2 to 6 & 2 to 8 \\
\hline
\end{tabular}

Table 7: $\beta=6.0$ IMCG $Z(3)$ string tensions and vortex densities for subsets of size $n_{g}$ out of 10 IMCG gauge copies, best MAG gauge copy

\begin{tabular}{||c|c|c||}
\hline$n_{g}$ & $\sigma_{Z(3)}$ & $p$ \\
\hline 2 & $0.033(1)$ & $2.73(4) \times 10^{-2}$ \\
\hline 4 & $0.030(1)$ & $2.67(4) \times 10^{-2}$ \\
\hline 6 & $0.029(1)$ & $2.64(4) \times 10^{-2}$ \\
\hline 8 & $0.029(1)$ & $2.62(4) \times 10^{-2}$ \\
\hline 10 & $0.029(1)$ & $2.61(4) \times 10^{-2}$ \\
\hline
\end{tabular}

Table 8: For cube dual to magnetic current link, $r_{m}(n) \equiv$ fraction of time $n$ faces are pierced by $Z(3)$ flux

\begin{tabular}{||c|c|c||}
\hline & $\beta=5.90$ & $\beta=6.0$ \\
\hline$r_{m}(0)$ & $0.14(1)$ & $0.16(1)$ \\
\hline$r_{m}(2)$ & $0.74(1)$ & $0.74(1)$ \\
\hline$r_{m}(3)$ & $0.09(2)$ & $0.07(2)$ \\
\hline$r_{m}(4)$ & $0.02(2)$ & $0.02(2)$ \\
\hline$r_{m}(5)$ & $0.002(2)$ & $0.001(1)$ \\
\hline$r_{m}(6)$ & $0.001(1)$ & $0.001(1)$ \\
\hline
\end{tabular}




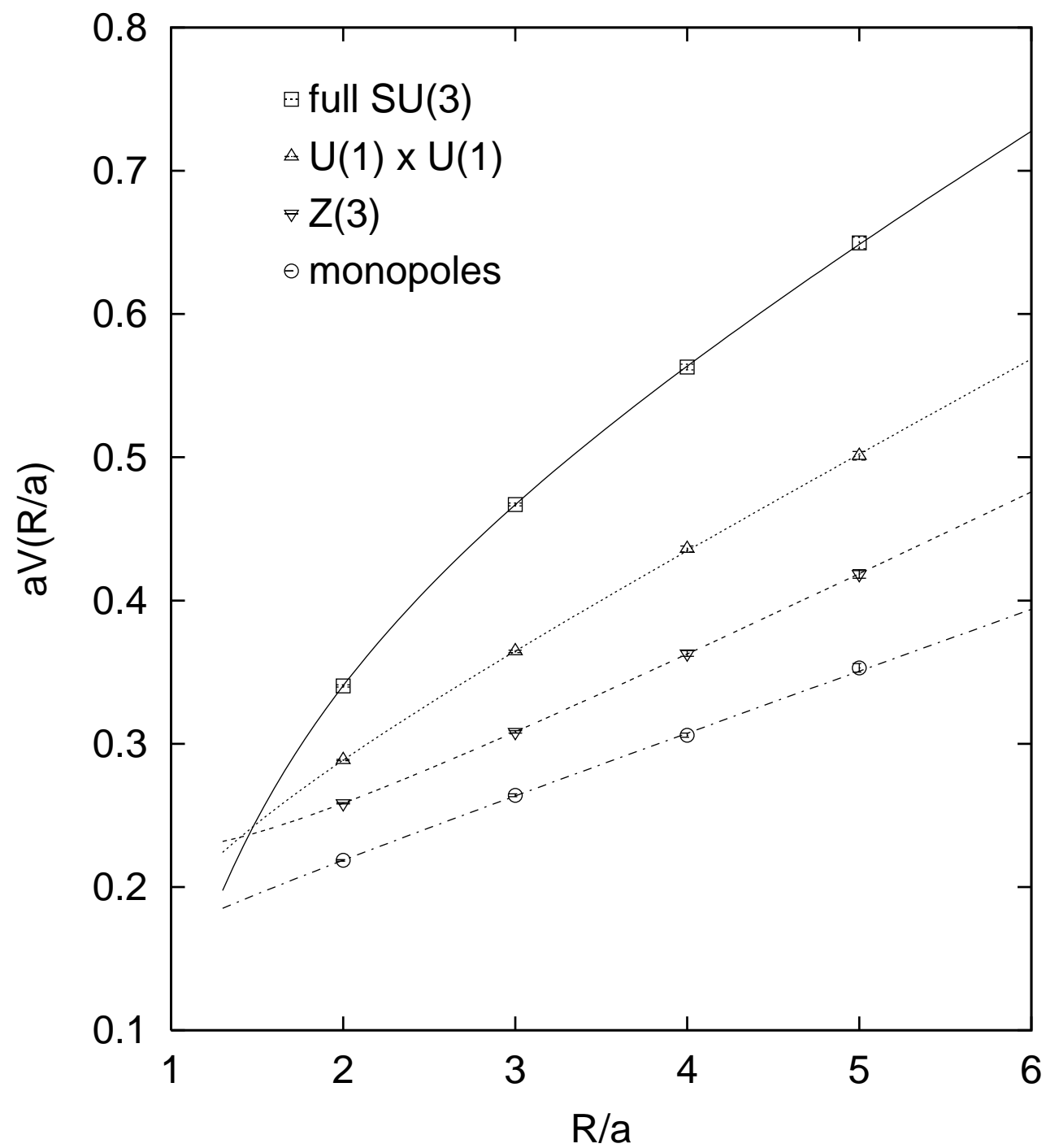

Figure 1: Heavy quark potentials at $\beta=5.9$ for full $S U(3), U(1) \times U(1), Z(3)$, and monopoles 


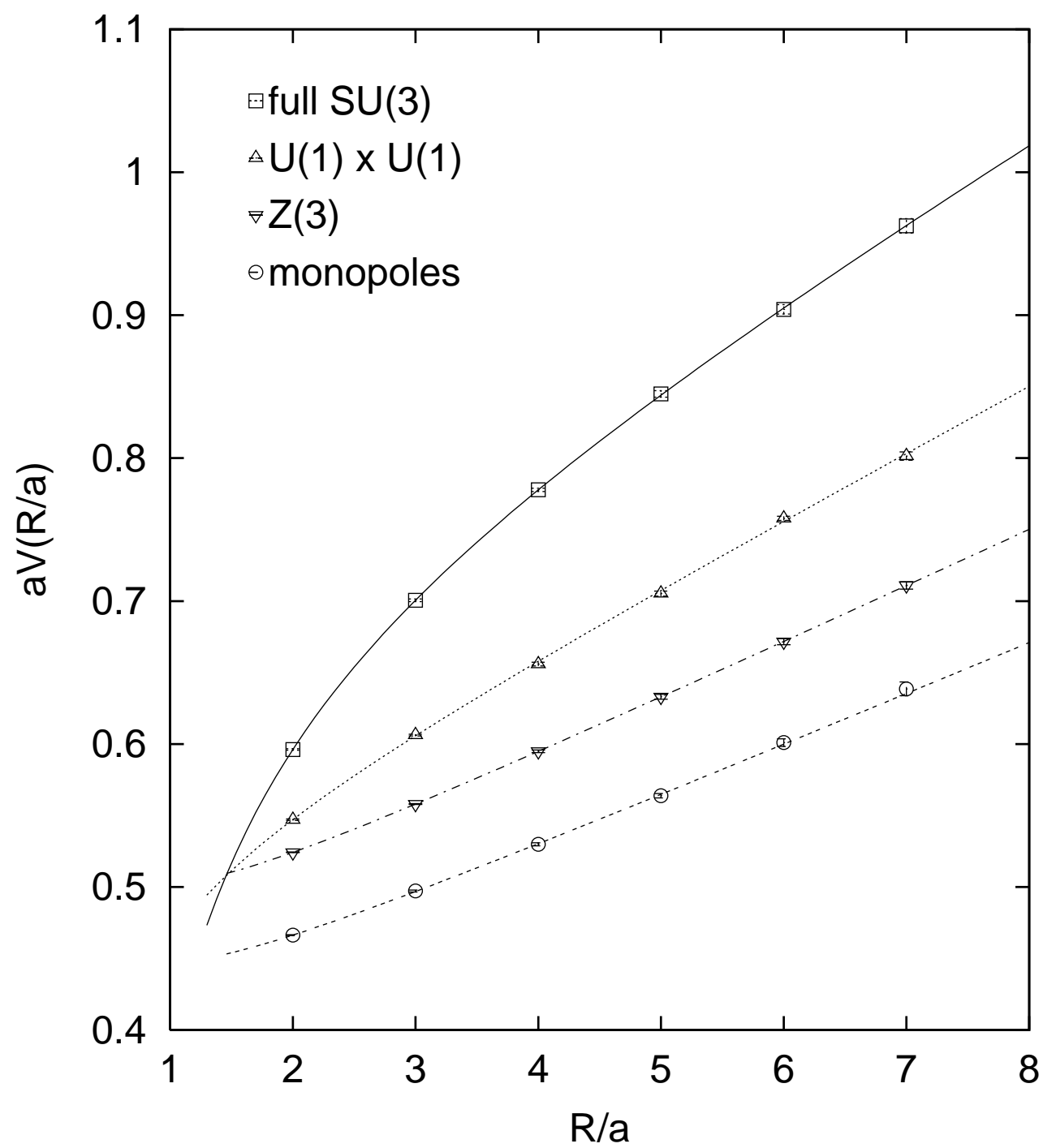

Figure 2: Heavy quark potentials at $\beta=6.0$ for full $S U(3), U(1) \times U(1), Z(3)$, and monopoles 